\title{
Intraoperative Neurophysiological Monitoring
}

\author{
Pablo MARTÍNEZ-SOLER ${ }^{1}$, Juan F MARTÍNEZ-CANCA ${ }^{2 *}$ and Miguel A SÁIZ-SEPÚLVEDA ${ }^{3}$ \\ ${ }^{1}$ Medical Student, University of Aberdeen, UK \\ ${ }^{2}$ Consultant Neurosurgeon, NEUROKONSILIA Inc, Spain \\ ${ }^{3}$ Consultant in Clinical Neurophysiology, NEUROKONSILIA Inc, Spain
}

*Corresponding author: Juan F Martínez-Canca, MD, MRCS, Consultant Neurosurgeon, Clinical Director Neurokonsilia Inc ${ }^{\mathrm{TM}}{ }^{\mathrm{C}}$., Málaga, Spain.
Received Date: March 08, 2021

Published Date: March 24, 2021

\section{What Is Intraoperative Neurophysiological Monitoring?}

Intraoperative neurophysiological monitoring (IONM) consists of the continuous analysis of the patients' neurological status using a set of tools and methods that allows us to assess the functional state of the neurological systems and pathways of interest. Different techniques, such as somatosensory evoked potentials (SSEPs), motor evoked potentials (MEPs), and others are employed according to the type of surgery and neurological tissue to be monitored.

\section{What is it Used For?}

The main goal of IONM is the live detection of any alteration in the monitored neurological tissue, thus allowing for a prompt intervention that will reduce any transient or permanent neurological damage and improve the surgical outcome as well as the overall quality of the medical treatment, including reduction in associated economic costs.

Monitoring allows the neurosurgeon to ponder variations in the surgical strategy in a dynamic way, based on the evolution of the surgery. Prognosis can also be predicted intraoperatively in some cases, optimising the choices regarding post-operative management and subsequent rehabilitation.

One of the aims of modern Medicine must be reducing the risk of post-operative deficit using as many means available as possible. In this regard, the use of IONM has proven its relevance in the different types of interventions it has been used in. Surgeons can now operate on pathologies previously out of reach due to the unacceptable risk without interoperative monitoring, as well as employing more aggressive techniques to achieve better results. This is why we can conclude IONM offers patients the best standard of care.

\section{When Should IONM Be Used?}

IONM is currently recommended in all surgical interventions where there could be potential risk of harm to any neurological structure.

\section{Neurophysiological Monitoring Techniques}

The neurophysiological technique of choice must yield adequate information in real time as well as being simple to use and sufficiently sensitive and specific regarding the particular risk in order to detect neurological insult on time.

There are two fundamental types of techniques in IONM: mapping techniques, used in the identification of structures during surgery, and monitoring techniques, which feeds real-time information about the functional integrity of the neurological structures during surgery.

Obviously, the latter will be more desirable, whenever possible, as it ensures immediate detection of surgical damage and improves the probability of correction.

\section{Mapping techniques:}

- Cortical mapping. 


\author{
- Subcortical mapping. \\ - Phase inversion of SSEPs. \\ - IV ventricle nuclei mapping. \\ - Dorsal columns mapping. \\ - Nerve root and pedicle screw mapping. \\ · Peripheral nerve mapping.
}

\section{Monitoring techniques:}

- Transcranial SSEP (t-SSEP).

- Transcranial MEP (t-PEM).

- Direct cortical stimulation MEP (c-MEP).

- Cortical SSEP (c-SSEP).

- Cortico-bulbar MEP.

- D waves.

- Reflexes (H reflex, Blink Reflex, bulbo-cavernous reflex, etc...).

- Brainstem auditory evoked potential (BAEP).

- Electromyography (EMG).

- Electroencephalogram (EEG).

The choice of technique is dependent on the type of surgery and neurological structures affected.

Somatosensory Evoked Potentials (SSEPs): Originally, IONM of the spinal cord was performed using SSEPs, gathering data on the functional state of the sensory pathway. SSEPs are obtained by electrical stimulation of mixed peripheral nerves in the upper and lower limbs, recording responses in the brain (through superficial or subdermal cranial electrodes) originated in the cortical sensory area. Propagation of SSEPs allows us to track its progression through the nerve, posterior roots, medial lemniscus, thalamus and cerebral cortex.

Motor Evoked Potential (MEP): IONM of the motor pathway is performed through Transcranial Electric Stimulation (TES), in which very brief electrical stimulations activate the central motor pathway and allow us to register motor responses at the spinal cord level (D-waves) or at the preferred muscle groups - depending on risk criteria - in the head, core, and limb regions. Stimulation may be continuous throughout the surgery or performed in stages where risk of harm to the nervous system is present, allowing a prompt detection of neurological insult and a swift initiation of the required protocol to avoid permanent neurological damage.

Continuous Electromyography Recording (CEMG): Allows detection of mechanically induced (traction, compression, etc...) neural activity which may indicate damage to motor neuron at the spinal or nerve root level. Especially relevant in spinal surgery due to its ability to promptly detect neurological insult in nerve roots and assess the integrity of nerve conduction between peripheral nerves and muscle tissue.

Stimulated Electromyography (SEMG): By applying electrical stimuli in surgeries where instrumentation is being inserted in the proximities of the spinal cord and nerve roots, functional integrity can be assessed to prevent radiculopathy and spinal cord damage. In the event of such damage, or a fissure/fracture of the vertebral pedicles, the surgeon can be alerted to reposition the prosthetics adequately in-situ.

Brainstem Auditory Evoked Potentials (BAEP): BAEPs are recorded via surface electrodes and the use of continuous auditory stimulation. Yields information about the state of the brainstem, hence mainly used in interventions where the region needs to be manipulated. The brainstem nuclei (grey matter) are more sensitive to ischaemia and surgical handling than the fibres (white matter): many components of BAEPs are generated in the brainstem nuclei and their successful recording depends on the indemnity of these structures. Components of BAEPs can be altered according to the level of the lesion, which makes them very useful to assess brainstem integrity during cerebello-pontine angle surgery as well as IV ventricle interventions.

Visually Evoked Potentials (VEP): Recorded using electrodes on the scalp and using brief flashes of light as a stimulus. Indicated for monitoring of interventions that pose a risk to the optic nerve.

Electro Encefalo Gram (EEG): Registers bioelectrical cerebral activity via subdermal cranial needles. Usually applied in diagnosis of cerebral pathology to ensure correct cerebral oxygenation during procedures (cerebrovascular surgery, carotid surgery, etc...) and to establish control in patients with a history of status epilepticus or a susceptibility to it.

Cortical and Subcortical Mapping: This technique allows precise location of functional areas of the cortex during supratentorial surgery to maintain them intact. The objective is to delimitate the functional areas before proceeding with tumoral extraction with adequate safety margins. In patients where the cortical language areas are involved, an awake surgery under sedation can be performed where the patient is dialogued with, providing feedback during the mapping process.

\section{Monitoring Surgery of the Spine and Spinal Cord}

Spinal surgery has seen an increase in numbers with the improvement of imaging techniques, surgical practice and a better understanding of the pathologies that affect the spine and spinal cord.

The possibility of undertaking a functional neurophysiological assessment during surgery became a reality in the 1970s with the use of SSEPs. The introduction of new techniques such as continuous EMG and the assessment of the corticospinal pathway via MEPs have improved the efficacy of intraoperative functional assessment. 
In the present day, multimodal IONM is routine practice in many spinal surgery centres.

Congenital malformations acquired spinal deformities and spinal tumours are contributors to the increase in pathologies associated to myelopathy. This is significant, as the precedent factor plays a role in the risk of damage to the spinal cord associated with surgery.

The use of multimodal IONM is a very effective method to assess the functional integrity of the spinal cord during spinal surgery. It improves postoperative results by reducing neurological deficits. Multimodal IONM has a sensitivity of $89 \%$ and a specificity of $99 \%$.

Various multi-centrestudies haveshownthatneurophysiological monitoring of spinal cord function during surgery reduces incidence of neurological damage up to $50 \%$.

Owing to its high sensitivity and specificity, IONM improves spinal surgery prognosis and is recommended in the following interventions:

- Correction of spinal deformities with scoliosis over $45^{\circ}$.

- Correction of congenital spinal anomalies.

- Resection of intra and extramedullary tumours.

- Extensive decompressions of cervical stenosis (both anterior and posterior approaches), as well as thoracic or lumbar stenosis that cause myelopathy or functional impediment of the cauda equina or individual nerve roots.

- $\quad$ Spinal arteriovenous malformations.

- $\quad$ Aortic lesions.

Experience accumulated in the past justifies the use of IONM as a routine procedure owing to its excellent efficiency avoiding neurological insult. Additionally, their use makes other currently routine tests obsolete, such as the waking test in scoliosis correction.

The use of vertebral pedicle screws has become common practice in many spine-stabilising surgical procedures. Its use in the lumbosacral region is well established and its implementation in the thoracic vertebrae is increasing steadily as it allows for a solid fixation of the spine. These screws must be inserted correctly into the vertebrae, respecting the integrity of the medial and lateral walls of the pedicle to achieve a correct position within the vertebral body. Breakage of the pedicle walls or vertebral body may be accompanied by an invasion of the nerve root exit foramina or the spinal canal. The screws are inserted "blindly" by the surgeon relying on his experience. The positioning is aided and checked through imaging techniques. Using IONM, continuous EMG combined with stimulating EMG applied through the screws themselves can ensure a correct insertion, as any inadequate positioning would be revealed by monitoring.
Different literature reviews place the incidence of cortical rupture between $5 \%$ and $40 \%$, depending on the type of screws used. Between $2 \%$ and $10 \%$ of the aforementioned will lead to refractory radiculopathy. IONM reduces the risk of radiculopathy to $1 \%$, another stunning demonstration of its potential for reducing postoperative deficit.

\section{Monitoring and Mapping of Cranial Nerves, Brainstem, and Peripheral Nerves}

Monitoring the activity of cranial nerves and peripheral nerves in skull base surgery reduces the risk of permanent postoperative neurological deficit. These are the most commonly monitored cranial nerves (in order of frequency):

- $\quad$ Facial nerve (cranial nerve VII).

- $\quad$ Recurrent nerve (branch of cranial nerve X).

- $\quad$ Lower cranial nerves (cranial nerves IX, X, XI and XII).

- $\quad$ Auditory nerve (cranial nerve VIII).

- Motor cranial nerves innervating the extrinsic ocular muscles (cranial nerves III, IV and VI).

IONM of these structures yields great advantages during skull base surgery, surgeries of the cerebello-pontine and in proximity of the jugular foramen. Additionally, these techniques are beneficial for monitoring of extracranial surgical manipulation, such as thoracic and thyroid surgery, carotid endarterectomies and any surgery involving the neck region, where the extracranial course of cranial nerves can be affected. IONM is also of great aid to the surgeon in treatment of hemifacial spasm and vestibular neurectomy, as well as in gaining access to the brainstem through the IV ventricle.

The principles of cranial nerve IONM are the electrical stimulation of the surgical field followed by the recording of motor potentials in the muscles innervated by the tested cranial nerve, thus confirming their anatomical location.

Furthermore, continuous EMG can immediately detect when the surgical technique has damaged a nerve.

\section{Monitored nerves}

A. Facial nerve (cranial nerve VII) branches have both an intracranial and extracranial course that mostly end their meandering in the face, where they are susceptible to damage. The there too; most notably due to its frequency, the removal of acoustic nerve tumours and vestibular schwannomas. These growths can very frequently involve the facial nerve as well as distort the anatomy of the area making it harder to identify the course of the nerve. The main goal of IONM of the facial nerve is to identify areas of the tumour that do not involve the nerve, in order to safely remove as much as possible without causing damage to CN VII.

Many intraoperative events can also signify a risk to the facial nerve: 
$\neg \quad$ The nerve can be lacerated directly during the surgical approach and dissection of the field.

$\neg \quad$ The use of instruments such as the electric scalpel, laser electrodes, or even a diamond drill, may create excessively high temperatures, producing thermal insult to the nerve.

$\neg \quad$ Iatrogenic lesions may also be produced by shearing forces on the axon during dissection, causing damages of varying grades to the structure itself or compromising its irrigation.

$\neg \quad$ Compression of the nerve is yet another mechanism of injury during surgery which must be avoided.

Injury to the facial nerve implies a loss of function of the facial muscles, which does not only cause cosmetic issues, but a wider scope of functional concerns such as inability to feed properly or close the eyes, leading to a myriad of related problems.

IONM of the facial nerve allows precise location of its course in the surgical field. Transcortical stimulation assesses the function of the nerve without interfering with the neurosurgeon's work. Additionally, it also allows detection of lesions or interactions with the nerve through continuous EMG, the latter producing prolonged muscle contractions.

For these reasons IONM of the facial nerve improves prognosis in surgeries that involve its manipulation.

Skull base surgery (meningiomas, epidermoid cysts, cholesteatomas, neurinomas, and other growths that may involve the nerve or distort it) can prove a challenging scenario for the identification and preservation of the facial nerve. Similarly, microvascular decompressions and pathologies of other cranial nerves (e.g., trigeminal neuralgias) may benefit from simultaneous IONM.

Surgical fields outside the cranium also pose risks to the facial nerve. Parotid gland tumour resections are the most frequent cause of facial nerve damage peripherally. The anatomy of the nerve and its multiple ramifications make identification of all the structures involved a key requirement for their preservation during surgery.

Facial trauma repair and temporomandibular joint surgeries are also worthy of mention when analysing surgical risk to the facial nerve.

B. Trigeminal nerve (cranial nerve V) can be monitored accurately in terms of motor function with similar techniques to those aforementioned with the facial nerve. EMG is taken from the muscles of mastication innervated by this nerve. The indications for IONM of CN V are similar to those of CN VII and they are frequently monitored together.

C. Oculomotor nerves (cranial nerves III, IV and VI) may be affected by tumours of the cavernous sinus. Lesion of these structures can be problematic, as they not only innervate the extrinsic muscles of the eye, but also carry autonomic fibres responsible for pupillary accommodation and change in its diameter. Monitoring of these nerves implies greater complexity as the electrodes recording EMG must be placed on intraorbital structures, requiring intricate access.

D. Glossopharyngeal nerve (cranial nerve IX) can be monitored by registering muscular activity of the soft palate.

E. Recurrent laryngeal nerve and superior laryngeal nerve (branches of cranial nerve $\mathrm{X}$ ) innervate laryngeal musculature where motor activity can be recorded following stimulation of the nerve in question. The recurrent laryngeal nerve is one of the most commonly injured structures during parotid and thyroid gland surgeries as well as anterior approaches to the cervical spine.

Routine identification of the recurrent laryngeal nerve during thyroid surgery has reduced the incidence of damage from $10 \%$ to $2 \%$. However, identification of the nerve have not been able to completely eliminate the incidence of long term effects following local damage, such as vocal cord paralysis and dysphagia, which arise from unilateral injury and dramatically affects the patients quality of life. Bilateral injury is significantly more dangerous and may result in death due to lethal airway compromise.

The difficulty in identifying these nerves is responsible for the high morbidity associated to these procedures, especially those with difficult surgical approaches. Classifying cases according to expected technical difficulty, the procedures considered high risk are reinterventions (previous subtotal thyroidectomies, hemithyroidectomies, and tumour recurrences), surgeries to treat extensive thyroid cancers, retro-sternal goitres, and toxic goitres.

IONM methodology includes the identification of the laryngeal nerves in the surgical field using the stimulation probe (mapping) as well as continuous monitoring techniques that assess the integrity of these structures without interfering with the surgical process.

These techniques are carried out by placing stimulating electrodes in the proximity of the nerves and measuring MEPs obtained by transcranial stimulation of the pyramidal tracts. In addition to the benefits of reduced morbidity, IONM provides resources for both investigations/diagnosis and a valuable aid in medicolegal proceedings.

a) As an instrument for investigations:

$\neg$ Provides useful data for understanding the electrophysiological and anatomical correlation of the recurrent laryngeal nerve.

$\neg \quad$ Evaluates prognosis during IONM.

b) Medico-legal advantages:

Reduces number of tracheostomies needed. 
$\neg \quad$ Registers and records intraoperative readings.

$\neg$ Aids in the development of standard procedures in surgery.

F. Accessory spinal nerve (cranial nerve XI) may be damaged fairly easily in cervical region interventions.

G. Hypoglossal nerve (cranial nerve XII) is responsible for innervation of the intrinsic tongue muscles. Bilateral lesion implies serious difficulties for swallowing and language. This small nerve must be monitored in surgeries that are close to the clivus region and the foramen magnum.

H. Auditory nerve (cranial nerve VIII) Is the most commonly monitored sensory cranial nerve. The risk of lesion is greatest in resection of tumours located in the cerebellopontine angle. It may be damaged by surgical handling or by electrocoagulation.

Brainstem auditory evoked potentials (BAET) are the most common form of monitorisation of this nerve. Another possibility would be to record the evoked potential directly on an exposed part of the nerve itself or on the surface of the cochlear nucleus. The last two methods rely on the adequate positioning of electrodes by the surgeon. Their use during the resection of vestibular Schwannomas contributes greatly to the preservation of auditory function.

I. Optic nerve (cranial nerve II) can be monitored through visually evoked potentials (VEPs) detected by electrodes placed on the scalp and stimulating the patient through brief flashes of light. This procedure is indicated in surgeries where there is a risk to the optic nerve.

J. Intraoperative mapping of the roof of the IV ventricle may aid the surgeon in locating safe entry points to the brainstem when resecting tumours or arteriovenous malformations (AVMs). Mapping is produced by recordings of EMGs in the muscles innervated by cranial nerves following stimulation of the ventricle roof. The importance of this technique resides in the ability to locate the nuclei of the cranial nerves when the anatomy of the area is distorted. Thanks to this procedure, resection of tumours from mesencephalon, protuberance, and bulbar regions is possible with a greater degree of safety by identifying "non-functional areas" and use them for surgical access, sparing cranial nerve nuclei from damage.

\section{Ionm In Supratentorial Regions}

Monitoring surgeries with SSEPs, EEGs, MEPs, etc has become mainstream procedure in many Neuroscience Centres for the treatment of intracranial aneurysms, AVMs, central nervous system tumours, and deep lesions of the insula.

IONM using SSEPs in supratentorial procedures chiefly assists in mapping the extent of lesions adjacent to the central sulcus and as a means of continuous monitoring during surgical treatment of AVMs. This last case is due to the tight relationship between cerebral irrigation and variations in SSEPs, especially useful when clamping or shunting vascular structures in the brain. Monitoring sites vary according to region of the aneurysm: Anterior Cerebral Artery and Anterior Communicating Artery aneurysms are more sensitive to lower limb SSEPs, whereas Middle Cerebral Artery aneurysms are more responsive to upper limb SSEPs. In the case of Carotid Artery aneurysms, both techniques may be valid depending on location of the lesion. Monitoring using SSEPs may also be useful in treatment of vascular pathologies close to central regions and those in proximity to somatosensory pathways. Recording SSEPs is key to performing proximal occlusion tests prior to embolization in order to assess the impact of blood flow restriction on healthy tissues. Regarding tumours, SSEPs are relegated to a secondary role in favour of monitoring motor pathways, which are deemed as priority in terms of morbidity. Additionally, SSEPs are sensitive to many other factors, such as raised ICP, distant bleeds, arterial pressure variations, body temperature, hypoxia, and metabolic changes.

Functional mapping using IONM is based on the use of SSEPs of the Median Nerve with direct cortical recording, seeking the "inversion phase" that allows identification of the central sulcus to indirectly locate the motor area of the cortex. In instances where the anatomy is extremely distorted by the lesion, mapping must be performed by direct cortical stimulation. SSEP recording and direct cortical stimulation are thus, complementary techniques that must be used synergistically to determine safe resection borders or transcortical entry points in the case of deeper surgeries, such as those involving the basal ganglia and internal capsule. The main purpose of IONM of the motor pathway in supratentorial scenarios is the preservation of motor function whilst achieving maximal resection.

\section{IONM With What Specialties?}

Many specialties may benefit from IONM:

๘ Neurosurgery: Supratentorial, brainstem, cerebellopontine angle, tumoral, AVM, myelopathic, and compressive pathologies of varied nature in the central and peripheral nervous system benefit from IONM techniques discussed previously

๘ Orthopaedics: Scoliosis, control of pedicle screw placement in vertebral fixations, congenital or acquired spinal malformations, vertebral lesions

๘ ENT: Cerebello-pontine angle tumours, vestibular tumours, parotid gland tumours, laryngeal pathology

$\varpi$ Interventional neuroradiology: Embolization of cerebral and medullary AVMs and aneurysms

ॠ Vascular surgery: Aortic surgery, carotid surgery 
๘ Maxillofacial: Cervical pathology, facial trauma, temporomandibular joint surgery

๘ Thoracic surgery: preventing injury to the recurrent laryngeal nerve

๘ General surgery: thyroidectomy, monitoring pelvic floor

\section{Cost-Effectiveness Analysis}

Unanimous opinion nowadays is that IONM is necessary due to its capacity to significantly prevent and/or attenuate neurological morbidity when performed by a Physician with full IONM competences. There is great evidence that the cost of IONM by no means approaches that of the sequels the patient will suffer if this technique is not practiced. Diverse estimations prove cost-effectiveness would be achieved by preventing paraplegia in as little as 1 patient out of 833. Additionally, some aspects of cost analysis are usually overlooked when studying the costs of permanent postoperative neurological deficits, such as the legal costs associated to these cases, compensations, adaptations of daily living environment, and, finally but not less importantly, human suffering. These factors are hard to account for but must be included to reveal the true cost-effectiveness of IONM. In the present day, use of motor pathway exploration techniques has noticeably increased reliability of IONM: there are no cases described as resulting in paraplegia with conserved MEPs during surgery. Published studies worldwide provide enough scientific evidence to recommend routine use of IONM in many types of surgical interventions across specialties.

\section{Acknowledgement}

None.

\section{Conflict of Interest}

No conflict of interest.

\section{References}

1. Bertani G (2009) Intraoperative mapping and monitoring of brain functions for the resection of low-grade gliomas: technical considerations. Neurosurgical focus 27(4): E4.

2. Calancie B (2014) Neuromonitoring with pulse-train stimulation for implantation of thoracic pedicle screws: a blinded and randomized clinical study. Part 1. Methods and alarm criteria. Journal of Neurosurgery: Spine 20(6): 675-691.

3. Deletis V, Fernandez-Conejero I, Ulkatan S, Peter Costantino (2009) Methodology for intraoperatively eliciting motor evoked potentials in the vocal muscles by electrical stimulation of the corticobulbar tract. Clinical Neurophysiology 120(2): 336-341.

4. Deletis V, Sala F (2008) Intraoperative neurophysiological monitoring of the spinal cord during spinal cord and spine surgery: a review focus on the corticospinal tracts. Clinical Neurophysiology 119(2): 248-264.
5. Charles C J Dong, David B Macdonald, Ryojo Akagami, Brian Westerberg, Ahmed Alkhani, et al. (2005) Intraoperative facial motor evoked potential monitoring with transcranial electrical stimulation during skull base surgery. Clinical Neurophysiology 116(3): 588-596.

6. Donohue ML, Viswaminathan Swaminathan, Jeremy L Gilbert, Charles W Fox, John Smale, et al. (2012) Intraoperative neuromonitoring: can the results of direct stimulation of titanium-alloy pedicle screws in the thoracic spine be trusted? Journal of Clinical Neurophysiology 29(6): 502-508.

7. Gonzalez AA, Dhiraj Jeyanandarajan, Chris Hansen, Gabriel Zada, Patrick C Hsieh (2009) Intraoperative neurophysiological monitoring during spine surgery: a review. Neurosurgical Focus 27(4): E6.

8. Gunnarsson T, Andrei V Krassioukov, Roger Sarjeant, Michael G Fehlings (2004) Real-time continuous intraoperative electromyographic and somatosensory evoked potential recordings in spinal surgery: correlation of clinical and electrophysiologic findings in a prospective, consecutive series of 213 cases. Spine 29(6): 677-684.

9. Guo L, Adrian W Gelb (2011) The use of motor evoked potential monitoring during cerebral aneurysm surgery to predict pure motor deficits due to subcortical ischemia. Clinical Neurophysiology 122(4): 648-655.

10. Holland NR (2002) Intraoperative electromyography. Journal of Clinical Neurophysiology 19(5): 444-453.

11. Macdonald DB, S Skinner, J Shils, C Yingling, American Society of Neurophysiological Monitoring (2013) Intraoperative motor evoked potential monitoring - A position statement by the American Society of Neurophysiological Monitoring. Clinical Neurophysiology 124(12): 2291-2316.

12. Macdonald DB (2006) Intraoperative motor evoked potential monitoring: overview and update. Journal of Clinical Neurophysiology and Computing 20(5): 347-377.

13. Parker SL, Anubhav G Amin, S Harrison Farber, Matthew J McGirt, Daniel M Sciubba, et al. (2011) Ability of electromyographic monitoring to determine the presence of malpositioned pedicle screws in the lumbosacral spine: analysis of 2450 consecutively placed screws. Journal of Neurosurgery: Spine 15(2): 130-135.

14.Simon MV (2013) Intraoperative neurophysiologic sensorimotor mapping and monitoring in supratentorial surgery. Journal of Clinical Neurophysiology 30(6): 571-590.

15. Simon MV (2011) Neurophysiologic intraoperative monitoring of the vestibulocochlear nerve. Journal of Clinical Neurophysiology 28(6): 566-581.

16. Thirumala PD (2011) Neurophysiologic intraoperative monitoring of olfactory and optic nerves. Journal of Clinical Neurophysiology 28(6): 538-542.

17. Ulkatan S, Milton Waner, Beatriz Arranz-Arranz, Inanna Weiss, Theresa MJO (2014) New methodology for facial nerve monitoring in extracranial surgeries of vascular malformations. Clinical Neurophysiology 125(4): 849-855. 